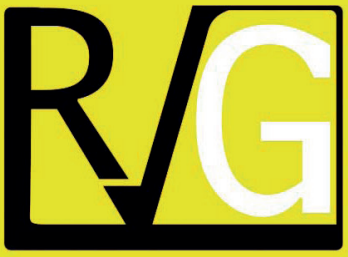

Julio - Septiembre, 2021

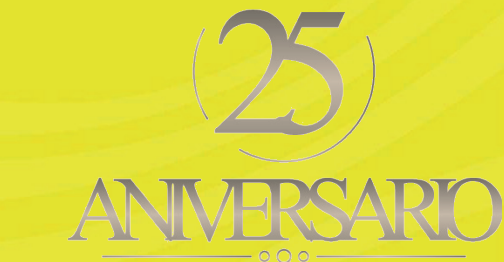

ANMERSARO
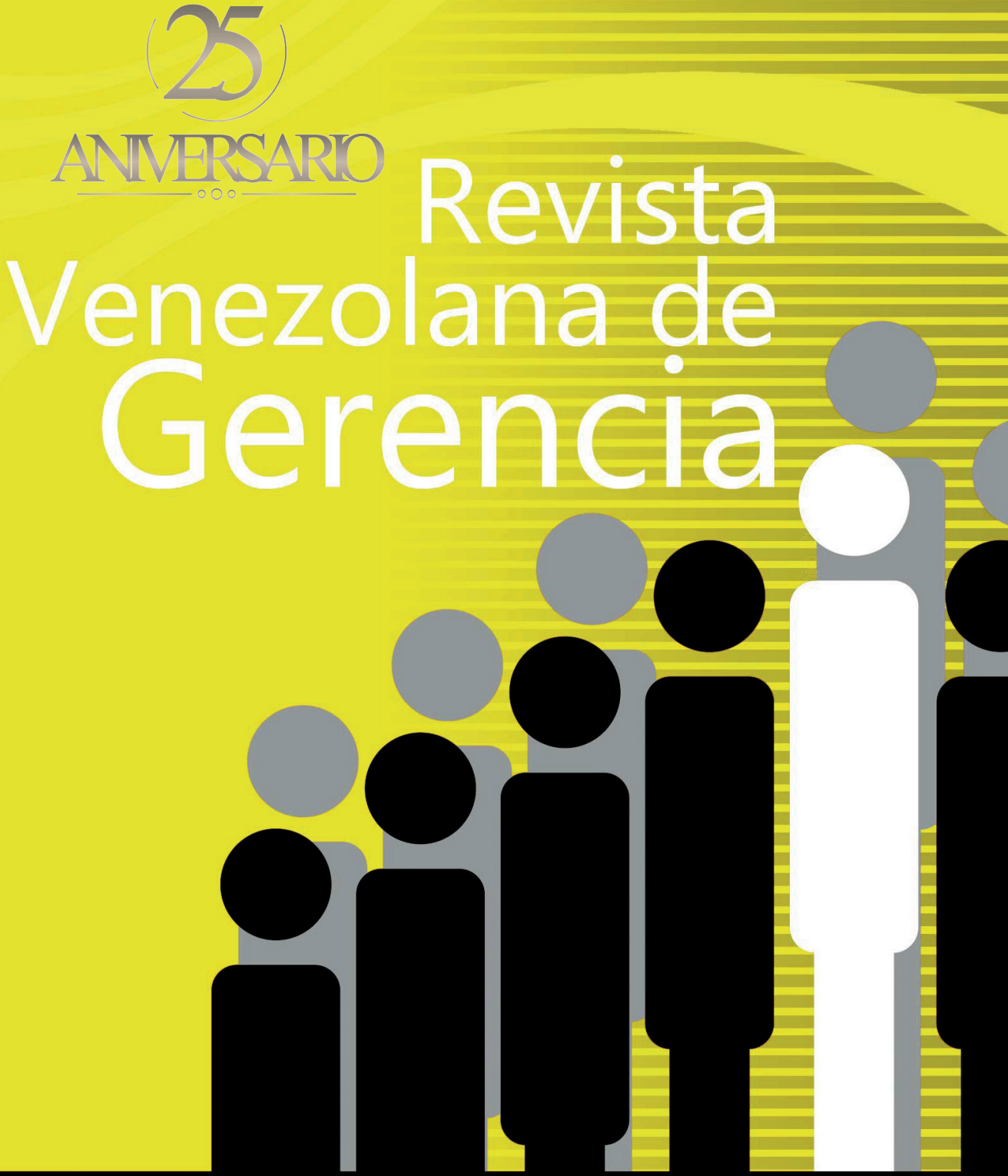

UNIVERSIDAD DEL ZULIA (LUZ)

Facultad de Ciencias Económicas y Sociales Centro de Estudios de la Empresa

ISSN 1315-99

Esta obra está bajo una licencia de Creative Comm Reconocimiento-NoComercial-Compartirlgual 3.0 Unpo http://creativecommons.org/licenses/by-nc-sa/3.0/deed.es 


\title{
Liderazgo y gestión empresarial: modelo de liderazgo global de cambio*
}

\author{
Peña-Acuña, Beatriz**
}

\section{Resumen}

La investigación tiene como objetivo describir y presentar un modelo acerca de liderazgo y gestión empresarial llamado "Liderazgo global de cambio". También presenta un cuestionario original validado derivado de este modelo. La metodología de investigación se basa en revisión bibliográfica especializada contando con una trayectoria extensa de investigación. Los resultados de este modelo apuntan a un líder capacitado por su visión global para generar el cambio en la cultura empresarial sabiendo dirigir a los empleados para que se co responsabilicen y se empoderen. Se concluye que el modelo de liderazgo, basado en factores humanos, es asequible en una sociedad tecnológica en la que la adaptación inteligente y creativa es más urgente.

Palabras clave: dignidad humana; participación; toma de decisiones; visión global; cambio

* Esta publicación ha sido posible, en parte, por la participación en el Proyecto Europeo Breed2Lead. Disruptive change agents: the new breed of leaders in Europe. breed2lead-project.eu/ Proyecto KA2 (Cooperation for Innovation and Exchange of Good practices) financiado por la Unión Europea.

** Doctora por la Universidad de Alicante - España. Honoris Causa por las universidades UCNE - EE. UU., UFA - Rusia y CUGS - México. Licenciada en Filología Hispánica. Licenciada en Periodismo. Posgraduada en Dirección y Producción en cine, vídeo y televisión. Profesora Titular de la Universidad de Huelva- España. beatriz.pa@dfilo.uhu.es, ORCID https://orcid.org/0000-0002-0951-795X 


\title{
Global twist leadership model
}

\begin{abstract}
This essay aims to describe and present a model about leadership and business management called "Global Twist Leadership." It also presents an original validated questionnaire derived from this model. The research methodology is based on a specialized bibliographic review, counting on a broad research trajectory. The results of this model point to a leader trained by his global vision to generate change in the business culture, knowing how to direct employees so that they take responsibility and empower themselves. We conclude that this leadership model, based on human factors, is affordable in a technological society in which intelligent and creative adaptation is more urgent.
\end{abstract}

Key words: human dignity; participation; decision making; global vision; change

\section{Introducción}

En este apartado se muestra una visión panorámica reciente de la investigación en satisfacción laboral con posturas controvertidas. También se detallan qué factores de este campo han influido quizás más para construir este modelo de liderazgo dejando otros enfoques de lado. La satisfacción personal en el desempeño del trabajo profesional dentro de una empresa supone de un proceso complejo del que forman parte prolijas variables (OsunaAlarcón y Rodríguez-Hernández, 2020) con importantes ramificaciones en instituciones no estrictamente empresariales (Ganga-Contreras et al. 2016).

Cascales (2010) distingue en una investigación entre los factores estructurales e individuales, y dentro de los individuales los objetivos y subjetivos. Los resultados significativos derivan de los factores individuales subjetivos, otorgándoles mayor importancia. En cambio, otra pesquisa empírica
(Crespí y Mascarilla, 2018) encuentra que el contenido del puesto de trabajo es el primer aspecto que valoran los empleados en su rango de satisfacción. García y Forero (2014) detectaron como motivos disposicionales de satisfacción en las que destacan de modo preferente el poder $o$ el reconocimiento. Otra indagación de Guzmán et al (2015) encuentra una correlación positiva significativa entre el empoderamiento y la satisfacción laboral.

Pujol-Cols y Davos (2018) señalan una insuficiencia de investigaciones que hayan estudiado de manera simultánea los efectos de determinantes disposicionales y situacionales sobre la satisfacción laboral. Sin embargo, se encuentra la revisión de estudios de Guerrero et al. (2018) que reconocen una fuerte relación entre liderazgo y satisfacción laboral. Martínez (2010) destaca como fenómeno negativo entre los empleados el "síndrome Burnout". Del mismo modo, Vega-Pérez et al. (2017) atiende los factores psicosociales 
de riesgo. Por fortuna en la actualidad las empresas obtienen mucha información para contrastar su situación percibida y sus objetivos (González y Rivera, 2020). De manera que conceptos como liderazgo, Responsabilidad Social Corporativa (RSC) o gestión pueden ser correctamente ponderados acorde a sus nuevas implicaciones, como las apuntadas por Peña-Acuña, Parra y Beltrán (2014) o las estudiadas a la luz del COVID-19 por Gonzálvez, Barrientos-Báez y Parra (2020).

Asimismo, se entiende primordial para este modelo analizar la literatura previa acerca del estilo de liderazgo en la gestión empresarial en sentido teórico (Botella, 2014). A la par se han tenido en cuenta los estudios empíricos que aportan un conocimiento aplicado (Rodríguez, 2010). Del mismo modo son destacables las propuestas más especializadas atendiendo además a la cultura empresarial nacional, como el caso de Venezuela realizado por Loaiza y Pirela (2015). Si cabe, se abordan otros enfoques desde la personalidad (Méndez, 2016).

También se enfatizan los modelos que plantean el liderazgo y la innovación a través de otras perspectivas (García, 2010; García, 2018) o con un enfoque feminista (Cuadrado, 2002; Barreros y Rodríguez, 2020). Ligada a esta temática, asimismo se ha indagado en la competencia socioemocional en la gestión empresarial (Loaiza Peña y Villacreses, 2018). Igualmente, ha tenido bastante peso otra cuestión derivada de los estilos de liderazgo como es la dirección de la comunicación empresarial (Mandiá-Rubal, 2019). En este campo se detecta, cómo repercute el estilo de liderazgo en la cultura empresarial (Hougaard y Carter, 2018) y, en concreto, en la comunicación interna (Gómez, 2020).

Así pues, para componer este modelo se ha partido de una metodología de revisión crítica de la literatura especializada, así como una trayectoria de indagación con metodología cuantitativa en muestras poblacionales y estudio de casos cualitativos empresariales con éxito. Ambos factores, han influido en analizar y sintetizar estos contenidos interdisciplinares teóricoprácticos de modo que, en este ensayo, se ha ideado este nuevo modelo de utilidad en el panorama de gerencial empresarial, objetivo de esta disertación.

En este modelo se conjugan términos con más trayectoria académica en el campo de los estilos de liderazgo como participación, toma de decisiones, cultura empresarial... En cambio, emergen otros conceptos socioculturales más novedosos como global, sociedad en cambio, empoderamiento y recursos TIC. Asimismo, se aborda desde otras perspectivas con elementos de la psicología organizacional (creatividad, habilidades socioemocionales, etc.) o de la comunicación empresarial (escucha, retroalimentación, etc.). Se atiende también a que el factor humano se integre con la tecnología (Zuckerman, 2017; Bustos Martínez et al. 2019).

Dichos estilos de liderazgo en la gestión empresarial se ven reflejados, por ello, en trabajos académicos que inciden en su función de marco de actuación. Sin ser exhaustivos, se enumeran estos estudios: Peña-Acuña, 2005 y 2010; Peña-Acuña et al. 2014; Sánchez, 2016, Ganga-Contreras, et al 2017 y PeñaAcuña 2017a. Ligadas a esta temática se encuentran las aportaciones de la competencia socioemocional en la gestión empresarial (Peña-Acuña y Del 
Henar, 2017c).

Todo lo anterior sirve para ponderar la dirección de la comunicación empresarial dentro del marco de la comunicación persuasiva, pero también como conformante sustancial de la realidad mercantil (Álvarez, 2010; MiraAgulló et al 2016; Peña, 2018a y 2018b y Peña, 2020). Todos estos trabajos citados ahondan en el estudio de cómo repercute el estilo de liderazgo en la cultura empresarial y en la comunicación interna (Peña et al. 2015; Peña et al. 2017b; Caldevilla, 2010; Mejías et al. 2019; Castro-Martínez y Díaz-Morilla, 2020). Se ha tenido en cuenta una revisión de la literatura especializada con metodología cuantitativa (Corona, 2018) en muestras poblacionales y estudio de casos cualitativos empresariales de éxito (Puche, 2020).

Se ha planteado habitualmente la componente nuclear de la comunicación empresarial como la enfocada a los públicos externos pero también se subraya, -tal como lo entienden Marín y García (2014)- que los públicos internos son básicos ya que la creación de figuras de liderazgo les afectan directamente aunque se considere que estas figuras son más propios del ciberactivismo en el espacio público ya que se basan en un manejo de valores transmisibles que son en mayor o menor grado aceptados por el público receptor al que va dirigido el mensaje y vehiculado por las nuevas tecnologías (Sierra-Caballero, 2018; Mancinas et al. 2018). Incluso hay investigaciones que relacionan la mejora de la imagen que los empleados poseen de su empresa a partir de las Relaciones públicas bidireccionales de ésta con el público que se convierte en financiador al sentirse involucrado con el proyecto y arrastra a los públicos internos (Felipe et al. 2018).

\section{Marco conceptual y descripción del modelo de liderazgo global de cambio (Global Twist Leadership)}

En este modelo el protagonismo lo ostentan tanto el líder como los empleados empoderados. Se plantean cuatro componentes acerca del líder. El primer factor de este modelo es acerca de la cualidad de visión global del líder acerca de los retos que acechan por las condiciones externas o internas a la empresa y la necesidad de cambios. Esta cualidad requiere que el líder se forme constantemente, busque información a través de herramientas adecuadas, como los big data, se deje aconsejar de forma externa cuando sea necesario de forma que adquiera conocimiento suficiente de los retos y contenga ideas claras relevantes al respecto, así como de la imagen positiva o negativa proyectada (Caldevilla et al. 2019).

Se trata de generar una cultura del consenso acerca de los problemas y una evaluación de los riesgos, de manera que se elijan las mejores herramientas de comunicación para lograr la participación y así se implementen las mejores soluciones posibles, algo que redunda en la motivación del equipo, como se desprende también de Maldonado Gómez de la Torre (2016) y Tavares Teixeira (2019).

El liderazgo global de cambio es un modelo de Liderazgo gerencial personal o liderazgo con visión global en el que este genera y consigue la participación activa de los empleados en la toma de decisiones hacia el cambio. En este modelo el protagonismo lo ostentan tanto el líder como los empleados empoderados (Alandette, 2011; Valle y García, 2019). Esta participación 
en consenso, con plena libertad, co responsabilidad y co empoderamiento está sustentada en la idea basilar de la dignidad humana sostenida por Aristóteles, expuesta en el corpus "Política" (Di Santo, 2009), así como en otros pensadores posteriores que se han ido sumando a esta concepción en la cultura occidental.

En este paradigma se plantean cuatro componentes acerca del líder a partir del análisis de otros modelos y autores. Asimismo, a través de componentes estudiados en investigaciones sucesivas acerca del liderazgo empresarial realizadas a lo largo de años con anterioridad. Por tanto, se desglosan a continuación estos componentes en cuatro subapartados de forma pormenorizada junto con los autores, modelos y estudios a través de los que se han colegido estos componentes.

\subsection{Visión global del líder}

El primer factor de este modelo es acerca de la cualidad de visión del líder (García-Morales et al. 2007) a la que se le añade el matiz de global (Wictor y Anderson, 2012; Clark, 2015; Mendelhall et al. 2016; Chhotray et al. 2018) teniendo en cuenta los retos que acechan por las condiciones externas o internas a la empresa y la necesidad de cambios (Pérez-Vallejo et al. 2017). Por un lado, esta cualidad requiere en la praxis que el dirigente entienda que se debe a una formación continua (Staat, 2018). Asimismo, este debe desarrollar habilidades de búsqueda seleccionando con atino información a través de herramientas digitales adecuadas (Del Brio y Peña, 2017) e implantando recursos digitales para la empresa como el uso de los big data para la toma de decisiones. Todos estos recursos sirven para captar el entorno que le rodea sabiendo analizar el contexto socioeconómico (Ruiz, Bodes y Dominicis, 2016) para disponer de esta visión.

Además, este modelo contiene implícitamente ciertas cualidades del dirigente que lo capacita para empujar y llevar hacia adelante proyectos. Entre estas cualidades se precisa que sepa escuchar activamente (Codina, 2004), que busque la retroalimentación acerca de los planteamientos antes de la toma de decisiones (Rodríguez del Tronco et al. 2018). Otro factor no menos importante es que se deje aconsejar con humildad (Thorndike, 2012). También es aconsejable que busque la consulta de forma externa, cuando sea necesario, de forma que el directivo adquiera conocimiento suficiente de los retos que presenta la empresa y contenga ideas claras y realistas relevantes al respecto.

Por otro lado, la visión global es asumida como aquella que trasciende más allá de la realidad práctica. Se caracteriza por la capacidad de análisis de varias condiciones relevantes externas e internas de la empresa que hacen necesarios la redirección de la toma de decisiones en sintonía hacia un giro de perspectiva (Christensen,1997). Esta reconducción plantea soluciones creativas y divergentes mayores para la dinámica interna y externa empresarial (Guilford, 1967).

\subsection{El líder planifica los procedimientos de participación y toma de decisiones}

El líder planifica los procedimientos eficaces para la toma de decisiones y 
estilo participativo tanto de directivos, mandos intermedios y empleados. Esto implica que el Ceo despliegue habilidades socioemocionales para influir y atraer a los demás hacia una causa (Carnegie, 1969; Goleman 1995, 2008). Igualmente sabe generar una cultura del consenso (Roberto, 2013) acerca de problemas y evaluación de los riesgos. Si cabe, este debe tener en cuenta que la votación en la toma de decisiones de los sujetos que participan y se implican, importa, es decir, tiene un valor constructivo (Sánchez, 2011) puesto que enriquece y es determinante para que la empresa progrese.

Después de la toma de decisiones, además, el directivo debe saber dirimir las mejores estrategias y herramientas de comunicación para lograr la participación del tejido empresarial y la implementación concreta de proyectos y soluciones (Naranjo, 2013). Es entonces cuando el tejido empresarial palpa y se conciencia de que se va construyendo la empresa con el esfuerzo común en la participación y toma de decisiones conjunta. De este modo, los agentes valoran el peso de estas dos acciones, la participación y la toma de decisiones, así como se empoderan y se co responsabilizan de forma progresiva de la corporación. Al mismo tiempo, se fortalecen y consolidan estos valores en la cultura empresarial (Coyle, 2018).

\subsection{Actitud positiva del líder frente a la toma de decisiones}

El líder despliega una actitud positiva (Rojas, 2013) frente a la toma de decisiones cimentada en el estilo participativo. Según Ferrer (2015) es importante que el líder tenga una visión propia positiva para que pueda extenderla a los demás, así como para la participación en el proceso de cambio por parte del tejido empresarial puesto que está asociado a la percepción positiva del mismo (Erwin y Garman, 2010) Además, los sujetos que muestran una mayor tendencia a la aceptación del cambio coinciden con que se adaptan mejor a las exigencias que los entornos laborales les imponen (Di Fabio y Gori, 2016).

Esta actitud positiva es conveniente que vaya reforzada de modo implícito a la actitud del optimismo y el engagement laboral (Maury-Ortiz et al. 2014). También que sea concomitante de forma subyacente a la cualidad de la flexibilidad y la adaptabilidad. Esta última capacidad implica subdimensiones como la curiosidad, la resiliencia (Ortunio, Magaly y Guevara, 2016), la gestión de la incertidumbre y habilidades para resolver problemas (RiquelmeCastaneda et al. 2020).

El cabeza confía en el refuerzo de la propia autoridad gracias al apoyo y la participación de otros en consenso. Asimismo, esta confianza está fundamentada en la experiencia de otros (Lockward, 2011). También en la potencia creativa colectiva de búsqueda de soluciones como estrategia de fondo de gestión del conocimiento (Córdoba et al. 2018). De este modo el líder sabe delegar (Chávez, 2013), frente a una actitud con afán controlador. A su vez, el dirigente muestra pericia para reforzar positivamente la toma de decisiones en consenso puesto que "los tomadores de decisiones pueden elegir las iniciativas con respecto a su responsabilidad corporativa proactivamente y estratégicamente" (Salgado et al. 2018: 128) 


\subsection{El dirigente genera cambio en la cultura empresarial}

El líder en definitiva genera cambio en la cultura empresarial (Chávez y Ibarra, 2016) creando valor estratégico (Rodríguez y Pedraja, 2009) dirigido a la visión global de partida (Gómez, 2008). Para ello el dirigente sabe involucrar a empleados (Guerrero et al. 2018), así como a muchas partes interesadas para que la toma de decisiones consensuada sea global (Rodríguez-Cruz y Pinto, 2017), eficaz (Rodríguez-Cruz, 2015) y motivadora. Además, el cabeza de empresa gestiona y extiende primero, una cultura de participación (Danet et al. 2014). Segundo, este transmite el cambio en los procesos (Seligman y Csikszentmihalyi, 2000). Tercero, este comunica visión global (Apolo et al. 2017) la cual, tras la experiencia en los procesos de participación, de co responsabilidad y de empoderamiento, acaban asimilando también los demás agentes del tejido empresarial.

Estos cuatro componentes del proceso que se suceden de forma concatenada en este modelo, se representan de forma ascendiente, (figura 1).

\section{Figura 1 \\ Esquema del Modelo de Liderazgo global de cambio (Global Twist Leadership)}

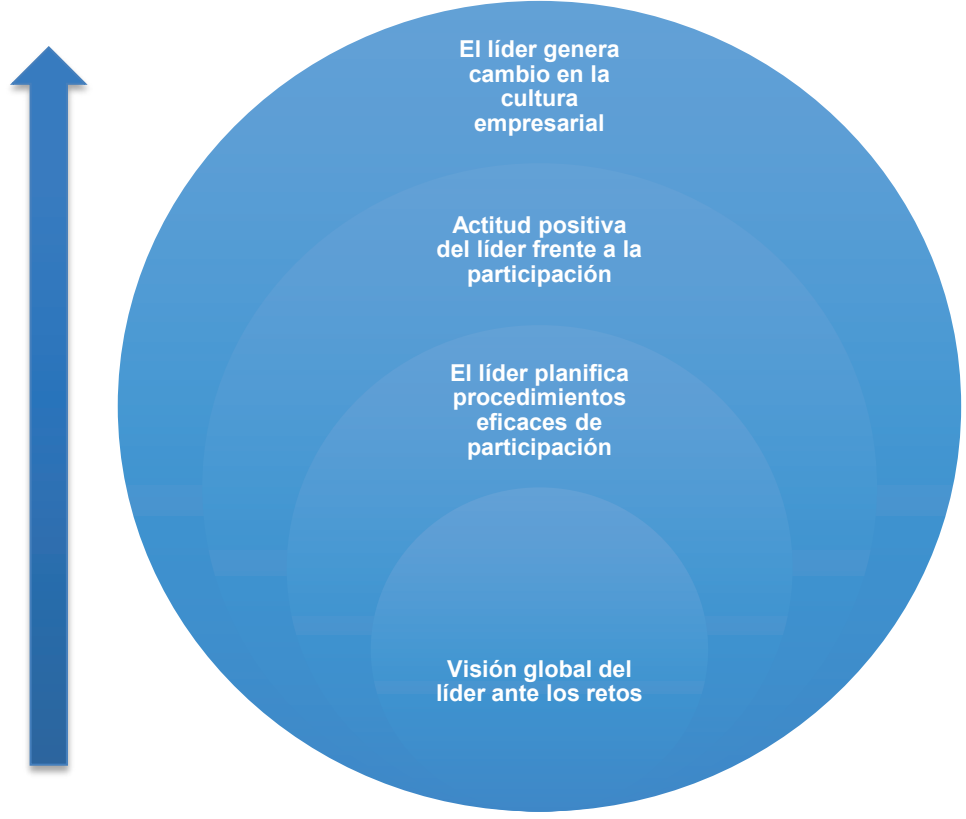

Fuente: elaboración propia 


\section{Cuestionario de Liderazgo global capacitado para el cambio (LGC)}

De este modelo expuesto con anterioridad se deriva un cuestionario compuesto de 24 preguntas llamado "Liderazgo global capacitado para el cambio (LGC)", que se ideó de forma original en 2017. Este test fue validado en castellano por treinta expertos.
Se adjunta a continuación el cuadro con los componentes (cuadro1) y después el test en sí (cuadro 2). Este es test al completo, preparado para extraer datos significativos acerca de estos componentes en una muestra significativa en un caso de empresa único o un caso múltiple de empresas. El cuestionario debe ser completado por los directivos.

\section{Cuadro 1}

\section{Componentes del test y preguntas ligadas a los componentes}

\begin{tabular}{lll}
\hline Componente & Visión global del líder acerca de los retos. & Preguntas \\
\hline Visión global & $\begin{array}{l}\text { Selección de procedimientos eficaces } \\
\text { dispuestos por el líder para la toma de } \\
\text { decisiones y estilo participativo. }\end{array}$ & $\begin{array}{l}\text { (preguntas 2, 6, 10, 14, 18, } \\
\text { 20, 22) }\end{array}$ \\
\hline Estrategias participativas & $\begin{array}{l}\text { Actitud positiva del líder frente a la toma de } \\
\text { decisiones basada en un estilo participativo }\end{array}$ & $\begin{array}{l}\text { (preguntas 3, 7, 11, 15, 19, } \\
21,23)\end{array}$ \\
\hline Actitud positiva & $\begin{array}{l}\text { El líder genera cambio en la cultura } \\
\text { empresarial dirigido a la visión global }\end{array}$ & (preguntas 4, 8, 12, 16, 24) \\
\hline Generación de cambio & &
\end{tabular}

Fuente: elaboración propia

El test de 24 ítems se contesta de forma gradual ascendente con cinco posibilidades (Cuadro 2, abajo; en columna a la derecha). Se presentan las preguntas en un cuadro sucesivamente en dos bloques.

\section{Cuadro 2}

\section{Cuestionario: Liderazgo global capacitado para el cambio (LGC)}

\section{Items o preguntas}

1. Tengo una visión global de los retos (nuevas tecnologías, innovación, tendencia, competencia, eficiencia, sostenibilidad, etc.) a los que se enfrenta la empresa y hacia esta dirección dirijo a mis empleados

2. La toma de decisiones es consensuada, una vez hemos decidido entre todos, el directivo y los empleados, cuál es la mejor

\section{Escala}

a) Nada en absoluto, b) Muy poco, c) A veces, d) A menudo, e) Muy a menudo.

a) Nada en absoluto, b) Muy poco, c) A veces, d) A menudo, e) Muy a menudo 


\title{
Cont... Cuadro 2
}

\begin{abstract}
3. Me hace sentir seguro tener el control final en la toma de decisiones

a) Nada en absoluto, b) Muy poco, c) A veces, d) A menudo, e) Muy a menudo.
\end{abstract}

4. Suelo comunicar que es necesario una cultura de cambio en los procesos para mejorar el rendimiento de la empresa y saber adaptarse a los nuevos tiempos.

a) Nada en absoluto, b) Muy poco, c) A veces, d) A menudo, e) Muy a menudo.

5. Recibo formación y busco información para adquirir una visión global de los retos (nuevas tecnologías, innovación, tendencia, competencia, eficiencia, etc.).

a) Nada en absoluto, b) Muy poco, c) A veces, d) A menudo, e) Muy a menudo.

6. Evalúo los riesgos asociados con cada alternativa que decidimos por consenso (junto con los empleados) antes de tomar una decisión entre a) Nada en absoluto, b) Muy poco, c) A todos.

veces, d) A menudo, e) Muy a menudo.

7. Dejar participar a los empleados en la toma de decisiones dirigido al cambio me da alegría, siento que no pierdo liderazgo, sino que refuerzo el liderazgo

8. Creo que involucrar a muchas partes interesadas para generar soluciones puede hacer que la toma de decisiones sea más eficaz y motivadora para dirigirnos a la cultura de la empresa y además a la a) Nada en absoluto, b) Muy poco, c) A veces, d) A menudo, e) Muy a menudo. visión dirigida al cambio.

9. Tengo una visión global de los retos (nuevas tecnologías, innovación, tendencia, competencia, eficiencia, etc.) y la comunico a mis empleados para que, entre ellos y yo, cambiemos los valores y la a) Nada en absoluto, b) Muy poco, c) A veces, d) A menudo, e) Muy a menudo. cultura empresarial lo que sea necesario

a) Nada en absoluto, b) Muy poco, c) A veces, d) A menudo, e) Muy a menudo.

10. Intentamos determinar en equipo, entre los empleados y yo, el problema real para realizar el cambio antes de comenzar un proceso de toma de decisiones.

a) Nada en absoluto, b) Muy poco, c) A veces, d) A menudo, e) Muy a menudo.

11. Me hace sentir más seguro contar y apoyarme en la visión de los empleados para la toma de decisiones dirigida al cambio, ellos están habituados a ello y me respetan como líder

12. Se comunicar y gestionar la participación de los trabajadores para que contribuyan en la toma de decisiones teniendo en cuenta la cultura de la empresa y además la visión dirigida al cambio.

a) Nada en absoluto, b) Muy poco, c) A veces, d) A menudo, e) Muy a menudo.

13. Hago uso el análisis de los big data (tanto de los datos adquiridos internamente como externamente) para la toma de decisiones para el cambio

a) Nada en absoluto, b) Muy poco, c) A veces, d) A menudo, e) Muy a menudo.

14. Si tengo dudas sobre la decisión consensuada, vuelvo a estudiar y a reunirme para verificar estas suposiciones con los empleados.

a) Nada en absoluto, b) Muy poco, c) A veces, d) A menudo, e) Muy a menudo.

a) Nada en absoluto, b) Muy poco, c) A veces, d) A menudo, e) Muy a menudo. 


\title{
Cont... Cuadro 2
}

\begin{abstract}
15. Entre los empleados y yo confiamos en nuestra propia experiencia para encontrar posibles soluciones a un nuevo reto que nos presenta el cambio.

a) Nada en absoluto, b) Muy poco, c) A veces, d) A menudo, e) Muy a menudo.
\end{abstract}

16. Delego en los trabajadores la toma de decisiones teniendo en cuenta que ellos tienen asimilada la cultura de la empresa y además la visión dirigida al cambio.

a) Nada en absoluto, b) Muy poco, c) A veces, d) A menudo, e) Muy a menudo.

17. Suelo contratar el análisis previo de una empresa Consultora que me permita tener una visión global al proceso de la toma de decisiones para el cambio

a) Nada en absoluto, b) Muy poco, c) A veces, d) A menudo, e) Muy a menudo.

18. Escucho a los demás para dirimir cuál es la mejor herramienta de comunicación de toma de decisiones para cada decisión específica dirigida a este cambio.

a) Nada en absoluto, b) Muy poco, c) A veces, d) A menudo, e) Muy a menudo.

19. En un proceso de consenso en la toma de decisiones, no me impongo, sino que tiendo a apoyar las propuestas de mis empleados y trato de encontrar formas de hacer que funcionen.

a) Nada en absoluto, b) Muy poco, c) A veces, d) A menudo, e) Muy a menudo.

20. Antes de comunicar la decisión consensuada, hemos creado un plan de implementación.

a) Nada en absoluto, b) Muy poco, c) $A$ veces, d) A menudo, e) Muy a menudo.

21. Fomento la innovación y la creatividad en la toma de decisiones que plantean y realizan los empleados.

a) Nada en absoluto, b) Muy poco, c) A veces, d) A menudo, e) Muy a menudo.

22. Determinamos los factores más importantes para tomar decisiones y luego usamos estos factores para evaluar estas elecciones consensuadas.

a) Nada en absoluto, b) Muy poco, c) A veces, d) A menudo, e) Muy a menudo

23. Hago hincapié en la confianza que tengo en la decisión consensuada como una forma de reforzar el apoyo que necesito de los demás para construir y llevar a cabo los planes de mejora.

a) Nada en absoluto, b) Muy poco, c) A veces, d) A menudo, e) Muy a menudo 24. Algunas de las opciones que hemos consensuado han supuesto un cambio de algún valor de la cultura de la empresa.

a) Nada en absoluto, b) Muy poco, c) A veces, d) A menudo, e) Muy a menudo

Fuente: elaboración propia

\section{Reflexiones finales}

La ciencia y la sociedad avanzan cuando se plantea una nueva visión o concepción acerca de la realidad.
Es necesario dar un paso adelante $y$ evolucionar hacia un estilo de liderazgo altamente participativo del empleado. El desarrollo de los individuos en el trabajo abarca una franja diaria amplia, supone 
una posibilidad de realización humana y responde, además, a la necesidad de supervivencia. Si cabe, hay que cuidar el factor humano con atención a su integración con la tecnología en una evolución empresarial que avanza hacia la ocupación laboral por parte de robots y programas informáticos, en la que se prioriza, según opinamos de forma miope, las ganancias. En cambio, si en el orden de prioridades las personas están en primer lugar en una empresa, si se enfoca el estilo de liderazgo adecuadamente, los sujetos acaban beneficiando a la empresa económicamente más y a sí mismas también para sobrevivir.

Se entiende que la sociedad está madura para dar ese paso de progreso tal y como propone este modelo gerencial en capacitar social a los líderes y a los individuos dotándolos de estrategias y herramientas propias de una cualidad humana que es la libertad que les permite participar, tomar decisiones teniendo una visión global. Con esta capacitación del individuo para socializar y organizarse con solidez es posible adaptarse al cambio y salir de la crisis que se le pueda presentar.

Este modelo con operatividad en el mundo empresarial está basado en factores humanos como la promoción de la dignidad humana de los individuos y las cualidades que ostentan, a saber, la participación, la toma de decisiones, la creatividad, la visión global y saber adaptarse al cambio son asequibles en una sociedad tecnológica. El impulso de la sociabilidad, la adaptación inteligente y creativa tanto de directivos como de empleados a través de estilos de liderazgo más avanzados se muestra más urgente hacia un bien común empresarial en medio de un panorama económico cambiante.

\section{Referencias bibliográficas}

Alandette, J. F. (2011). Empoderamiento en los sectores comercial y de servicios. Pensamiento psicológico, 9(16), 27-40. https://revistas. javerianacali.edu.co/index.php/ pensamientopsicologico/article/ view/163

Álvarez García, A. M. (2010), Principales efectos y ventajas del uso de las nuevas tecnologías en campañas electorales. CIC. Cuadernos De Información y Comunicación, 15, 5584. https://revistas.ucm.es/index.php/ CIYC/article/view/CIYC1010110055A

Apolo, D., Báez, V., Pauker, L., y Pasquel, G. (2017). Gestión de Comunicación Corporativa: consideraciones para el abordaje de su estudio y práctica. Revista Latina de Comunicación Social, 72, 521-539. https://doi. org/10.4185/RLCS-2017-1177

Barreros Gutiérrez, Y. y Rodríguez Agudín, C. (2020). Innovación y estilos de liderazgo. Capital humano: revista para la integración y desarrollo de los recursos humanos, 351. https://dialnet.unirioja.es/ servlet/articulo?codigo $=7477914$

Botella Carrubi, M. D. (2014). La evolución en los estilos de liderazgo y su impacto en las dimensiones de compromiso organizativo. Tesis doctoral. Universidad Católica de Valencia San Vicente Mártir, Valencia, España.

Bustos Martínez, L., De Santiago Ortega, P. P., Martínez Miró, M. A., y Rengifo Hidalgo, M. S. (2019). Discursos de odio: una epidemia que se propaga en la red. Estado de la cuestión sobre el racismo y la xenofobia en las redes sociales. Mediaciones Sociales, 18, 25-42. https://doi.org/10.5209/ $\underline{\text { meso.64527 }}$

Caldevilla Domínguez, D. (2010). La 
cara interna de la comunicación en la empresa. Visión Libros

Caldevilla Domínguez, D., Rodríguez Terceño, J., y Barrientos Báez, A. (2019). El malestar social a través de las nuevas tecnologías: Twitter como herramienta política. Revista latina de comunicación social, 74, 12641290. https://doi.org/10.4185/RLCS2019-1383-66

Carnegie, D. (1964). How to Win Friends and Influence People. Simon and Schuster.

Cascales Mira, M. (2010). Análisis de la satisfacción laboral en España. Documentos de trabajo, 2(5), 1-41. https://dialnet.unirioja.es/servlet/ articulo?codigo $=5708605$

Castro-Martínez, A. y Díaz-Morilla, P. (2020). Comunicación interna y gestión de bienestar y felicidad en la empresa española. El Profesional de la información, 29(3), 290-324. https://doi.org/10.3145/epi.2020. may.24

Chávez Donoso, S. (2013). La práctica del liderazgo en la empresa de hoy: Cómo el liderazgo puede incidir sobre las condiciones de seguridad y la eficiencia de los procesos de gestión organizacional. Ciencias Psicológicas, 7(1), 69-81. https://doi. org/10.22235/cp.v7i1.40

Chávez Martínez, J. J. e Ibarra Michel, J. P. (2016). Liderazgo y cambio cultural en la organización para la sustentabilidad. Telos, 18(1), 138-158. https://www.redalyc.org/ pdf/993/99344833009.pdf

Chhotray, S., Sivertsson, O., \& Tell, J. (2018). The roles of Leadership, Vision and Empowerment in Born Global Companies. Journal of International Entrepreneurship, 16, 38-57. https://doi.org/10.1007/ s10843-017-0201-8
Christensen, Clayton M. (1992). The innovator's dilemma: the revolutionary book that will change the way you do business. Harper Business.

Clark, J. (2015). Five principles of Global Leadership: How to Manage the Complexities of Global Leadership. Westbow Press.

Codina Jiménez, A. (2004). Saber escuchar. Un intangible valioso. Intangible capital, 3, 1-24. https:// www.intangiblecapital.org/index.php/ ic/article/view/23

Córdoba Zuñiga, E., Castillo Toro, J. L., y Castillo Quesada, N. (2018). Creatividad e innovación: Motores de desarrollo empresarial. Lámpsakos, 19, 55-65.

Corona Lisboa, J. L. (2018). Investigación cualitativa: fundamentos epistemológicos, teóricos y metodológicos. Vivat Academia, Revista de Comunicación, 144, 69-76. http://doi.org/10.15178/ va.2018.144.69-76

Coyle, D. (2018). The Culture Code: The Secrets of Highly Successful Groups. Bantam.

Crespí Vallbona, M. y Mascarilla Miró, O. (2018). Satisfacción laboral. El caso de los empleados del sector de las tecnologías de la información en España. Universia Business Review, 58, 36-51. https://doi.org/10.3232/ UBR.2018.V15.N2.02

Cuadrado Guirao, M. I. (2002). Estilos de liderazgo y género: una perspectiva psicosocial. Tesis doctoral. Universidad de Almería, Almería, España.

Danet, A., March, J. C. y García Romera, I. (2014). Comunicación, participación y liderazgo en la percepción del clima emocional en 
un hospital universitario de Andalucía -España-. Caderno de Saúde Pública, 30(3), 546-558 https://doi. org/10.1590/0102-311X00167712

Del Brio, N. y Peña Ceñiga, R. (2017). Liderazgo digital: los líderes también se transforman. Observatorio de recursos humanos y relaciones laborales, 125, 12-14. https://dialnet.unirioja.es/servlet/ articulo?codigo $=6062307$

Di Fabio, A. \& Gori, A. (2016). Developing a New Instrument for Assessing Acceptance of Change. Frontiers of Psychology, 7, 1-10. https://doi. org/10.3389/fpsyg.2016.00802

Di Santo, L. (2009). Filosofía de la paz y la Dignidad Humana. Frónesis, 16(2), 233-248. https://biblat.unam.mx/es/ revista/fronesis-maracaibo/articulo/ filosofia-de-la-paz-y-la-dignidadhumana

Erwin, D. \& Garman,A. (2010). Resistance to organizational change: linking research and practice. Leadership \& Organization Development Journal, 31(1), 39-56. http://doi. org/10.1108/01437731011010371

Felipe Morales, A., Caldevilla Domínguez, D., Rodríguez Terceño, J., y Del Valle Mejías, M. E. (2018). Crowdfunding: comunicación del mecenazgo 2.0: filantropía digital en las relaciones públicas bidireccionales. Revista de Ciencias de la Comunicación e Información, 23(1), 29-41. http://doi. org/10.35742/rcci.2018.23(1).29-41

Ferrer Davalos, R. M. (2015). La influencia del factor humano, el liderazgo y la cultura de las organizaciones en los procesos de implementación y gestión del cambio organizacional. Revista Internacional de Investigación en Ciencias Sociales, 11(1),102-114. https://dialnet.unirioja.es/servlet/ articulo?codigo $=5156083$
Ganga-Contreras, F., Navarrete, E., y Suárez, W. (2017). Aproximación a los fundamentos teóricos de liderazgo auténtico. Revista Venezolana de Gerencia, 22 (77), 36-55. http:// docente.ulagos.cl/franciscoganga/ index.php/2017/07/12/aproximaciona-los-fundamentos-teoricos-delliderazgo-autentico/

Ganga-Contreras, F., Villegas, F., Urriola, K. y Tapia, M. (2016). Gestión de núcleos investigativos e impacto en la generación de nuevo conocimiento: el caso de la Universidad de Antofagasta de Chile. Revista Venezolana de Gerencia, 21 (75), 382-410. http:// docente.ulagos.cl/franciscoganga/ index.php/2016/12/14/gestion-denucleos-investigativos-caso-de-launiversidad-de-antofagasta-de-chile/

García Echeverría, S. (2010). Innovación y liderazgo en nuevos estilos de dirección empresarial. Madrid: Instituto de Dirección y Organización de Empresas.

García Manjón, J. V. (2018). Relación entre estilos de liderazgo e innovación corporativa. Relación entre estilos de liderazgo e innovación corporativa. Trabajo: Revista iberoamericana de relaciones laborales, 36, 5767. https://www.researchgate.net/ publication/332856653 Relacion entre liderazgo e innovacion en las pymes de la comuna once de Medellin

García Morales, V. J., Romerosa Martínez, M. M. y Llorens Montes, F. J. (2007). Liderazgo transformacional: influencia en la visión compartida, aprendizaje, innovación y resultado organizativo. Revista europea de dirección y economía de la empresa, 16 (4), 25-46. https://dialnet.unirioja. $\underline{\text { es/servlet/articulo?codigo }=2504888}$

García Rubiano, M. y Forero Aponte, 
Liderazgo y gestión empresarial: modelo de liderazgo global de cambio

C. (2014). Motivación y satisfacción laborales como facilitadores del cambio organizacional. Una explicación desde las ecuaciones estructurales. Psicoagente, 17(31), 120-142.

Goleman, D. (1995). Inteligencia emocional. Editorial Kairos.

Goleman, D. (2008). Inteligencia social. Barcelona: Editorial Kairos.

Gómez Ortiz, R. A. (2008). El liderazgo empresarial para la innovación tecnológica en las micro, pequeñas y medianas empresas. Pensamiento y gestión, 24, 158-194.

Gómez, C. (2020). Progreso en Comunicación interna: qué ha cambiado en los últimos años. Capital humano: revista para la integración y desarrollo de los recursos humanos, 357

González Flores, I., y Rivera Riquenes, J. (2020). Auditoría 2.0, una perspectiva para su ejecución en el entorno empresarial utilizando técnicas de minería de procesos. Vivat Academia, Revista de Comunicación, 150, 47-57. http://doi. org/10.15178/va.2020.150.47-57

Gonzálvez Vallés, J. E., BarrientosBáez, A. y Parra López, E. (2020). Gobernanza y comunicación en la Universidad. Estudio a partir del COVID-19. Revista Venezolana de Gerencia, 25 (91), 1247-1261. https://www.researchgate.net/ publication/28199978 Liderazgo transformacional influencia en la vision compartida aprendizaje innovacion y resultado organizativo

Guerrero Bejarano, M. A., Añazco Camacho, K. A., Valdivieso Salas, R. P. y Sánchez Córdova, O. W. (2018). Los Estilos de Liderazgo y su efecto en la Satisfacción Laboral. INNOVA Research Journal, 3 (10), 142-148. https://doi.org/10.33890/innova. v3.n10.2018.908

Guilford, J. P. (1967). The nature of Human Intelligence. McGrawHill.

Guzmán Delfino, C. P., Pontes Macarulla, P. y Szuflita, M. (2015). Empowerment y satisfacción laboral. ReiDoCrea, 4, 66-73. https://digibug. ugr.es/handle/10481/36048

Hougaard, R. \& Carter, J. (2018). The mind of the leader. How to lead yourself, your people and your organization for extraordinary results. Harvard Business Review Press.

Loaiza Massuh, E. M. L., Peña Cabrera, G. y Villacreses Cobo, M. (2018). Implicancias de la Inteligencia Emocional en los Estilos de Liderazgo. RECIMUNDO: Revista Científica de la Investigación y el Conocimiento, 2 (1), 724-733. https://editorial.ucsg. edu.ec/ojs-alternativas/index.php/ alternativas-ucsg/article/view/120

Loaiza, C. T. y Pirela, L. (2015). Liderazgo en las organizaciones venezolanas. Revista Venezolana de Gerencia, 69, 152 - 171. https://www.redalyc.org/ pdf/290/29036968009.pdf

Lockward Dargam, A. M. (2011). El rol de la confianza en las organizaciones a través de los distintos enfoques o pensamientos de la administración. Ciencia y Sociedad, 36 (3), 464-502. https://doi.org/10.22206/CYS.2011. V36I3.PP464-502

Maldonado Gómez De La Torre, A. E. (2016). Contribuciones transmetodológicas para el análisis de procesos comunicativos contemporáneos, Mediaciones Sociales, 15, 1-15. https://doi. org/10.5209/MESO.54540

Mancinas Chávez, R., MorenoCabezudo, J. A., y Ruiz-Alba, N. (2019). Liderazgo de la prensa nativa 
digital frente a la prensa de referencia en España. El caso Cifuentes en Eldiario.es, El Confidencial, El País y El Mundo. Revista Latina de Comunicación Social, 74, 14341451. https://doi.org/10.4185/RLCS2019-1392

Mandiá-Rubal, S. (2019). Presencia y nivel de actividad en redes científicas de los investigadores españoles que publican en revistas de Comunicación indexadas en Scopus (Elsevier). Tesis doctoral. Universidad de Santiago de Compostela, Santiago, España.

Marín Dueñas, P., y García García, M. (2014). El estudio de la comunicación interna en el contexto del Grado de publicidad y RR.PP. de la universidad de Cádiz. Historia y Comunicación Social, 18, 97-108. https://doi. org/10.5209/rev HICS.2013. v18.43951

Martínez Pérez, A. (2010). El síndrome de Burnout. Evolución conceptual y estado actual de la cuestión. Revista de Comunicación Vivat Academia, 112, 42-80. https://www.redalyc.org/ pdf/5257/525752962004.pdf

Maury-Ortiz, J. G., Martínez-Lugo, Miguel E., y González-Colón, Z. L. (2014). Relación del optimismo, la personalidad resistente y el engagement con el trabajo en una muestra de empleados. Revista Puertorriqueña de Psicología, 25(2), 280-296. https://www.redalyc.org/ articulo.oa?id=233245622008

Mejías Acosta, A., Santelices Malfani, I., Galleguillos Peralta, L. y D’Armas Regnault, M. (2019). Perfil del liderazgo emprendedor del estudiante de ingeniería industrial: un estudio exploratorio en el contexto venezolano. Revista Inclusiones, 6(1), 53-75.

Mendelhall, M. E., LI, Ming \& Osland,
J. S. (2016). Five years of global leadership research, 2010-2014: Patterns, themes and future directions. In Joyce S. Osland, LI Ming \& Mark E. Mendelhall (Eds.). Advances in Global Leadership, 9, Bingley: Emerald Group Publishing Limited.

Méndez Bravo, J. César (2016). La influencia de la personalidad en los estilos de liderazgo. INNOVA Research Journal, 1(12), 52-58. https://bit.ly/3dUzGEh

Mira-Agulló, J. G., Peña-Acuña, B. y Parra-Meroño, M. C. (2016). La imagen del CEO: Estrategia de comunicación corporativa. Opción. 31 (3), 244-266. https://www.redalyc. org/pdf/310/31048901013.pdf

Naranjo Rivera, O. (2013). Diseño del plan de comunicación para la unidad de emprendimiento de la corporación universitaria adventista de Colombia. Revista Ciencias Estratégicas, 21 (29), 153-175. https://repository.upb. edu.co/handle/20.500.11912/7715

Ortunio C., Magaly S. y Guevara, H. R. (2016). Aproximación teórica al constructo resiliencia. Comunidad y Salud, 14(2), 96-105. http://www. redalyc.org/pdf/3757/375749517012. pdf

Osuna-Alarcón, M. R. y RodríguezHernández, M. P. (2020). Investigación, desarrollo e innovación en el sector empresarial español: dificultades para su implementación. El profesional de la información, 29(1), 290-319. https:// doi.org/10.3145/epi.2020.ene. 19

Peña-Acuña, B. (2005). Dirección de comunicación y habilidades directivas. Revista de la Comunicación de la SEECl, 12, 36-54. $\quad$ https://doi.org/10.15198/ seeci.2005.12.36-54 
Liderazgo y gestión empresarial: modelo de liderazgo global de cambio

Peña-Acuña, B. (2010). El liderazgo de la protagonista en Anatomía de Grey. Vivat Academia, Revista de Comunicación, 110, 35-48. https:// doi.org/10.15178/va.2010.110.35-48

Peña-Acuña, B. (2017a). Critical revision of leadership styles in management and company cases. Zagreb: Intechopen.

Peña-Acuña, B. (2018b). Los big data en la dirección de comunicación digital. En Caldevilla Domínguez, D. (Coord.). Perfiles actuales de la información y en los informadores. Tecnos

Peña-Acuña, B. (coord.) (2018a). Digital Communication Management. Zagreb: Intechopen.

Peña-Acuña, B. y Del Henar SánchezCobarro, P. (2017c). Socio-emotional skills and business communication. Procedia Social and Behavioral Sciences, 237, 914-918. https://doi. org/10.1016/j.sbspro.2017.02.128

Peña-Acuña, B., Del Henar-SánchezCobarro, P. y Wandosell-Fernández De Bobadilla, G. (2015). Estrategias inteligentes de comunicación interna y gestión empresarial. Opción: Revista de Ciencias Sociales y Humanas. 31 (3), 44-966. https://www.redalyc.org/ pdf/310/31045567050.pdf

Peña-Acuña, B., Parra Meroño, M. C. y Beltrán Bueno, M. A. (2014). Liderazgo resonante. Un análisis a través de grupos de discusión. Historia y Comunicación Social, 19, 143-151. https://doi. org/10.5209/rev HICS.2014. v19.44947

Peña-Acuña, B.; Caldevilla-Domínguez, D. y Batalla-Navarro, P. (2017b). Tres casos de empresas internacionales con éxito: estudio de las estrategias de comunicación interna. Chasqui. Revista Latinoamericana de
Comunicación, 315-330. https:// revistachasqui.org/index.php/ chasqui/article/view/2697

Pérez-Vallejo, L. M., Vilariño-Corella, C. M. y Ronda-Pupo, G. A. (2017). El cambio organizacional como herramienta para coadyuvar con la implementación de la estrategia. Ingeniería Industrial, 38(3), 323-332. https://rii.cujae.edu.cu/index.php/ revistaind/article/view/605

Puche Bazarra, W. (2020). ¿Innovadoramente creativos o creativamente innovadores? Gerencia y gestión cultural en tiempos de economía naranja. Revista Inclusiones, 7, 85-109. www. archivosrevistainclusiones.com/ gallery $/ 6 \% 20 \mathrm{vol} \% 207 \% 20$ num $\% 20$ espcolombiacaribe\%20inclusiones. $\underline{\mathrm{pdf}}$

Pujol-Cols, L. J. y Davos, G. E. (2018). Satisfacción laboral: una revisión de la literatura acerca de sus principales determinantes. Estudios Gerenciales. Journal of Management and Economics for Iberoamerica, 34(146), 3-18. https://doi. org/10.18046/j.estger.2018.146.2809

Riquelme-Castaneda, J. A., PedrajaRejas, L. M. y Vega-Masso, R. A. (2020). El liderazgo y la gestión en la solución de problemas perversos. Una revisión de la literatura. Formación Universitaria, 13 (1), 135-144. http://dx.doi.org/10.4067/ $\underline{\mathrm{S} 0718-50062020000100135}$

Roberto, M. A. (2013). Why Great Leaders Don't Take Yes for an Answer: Managing for Conflict and Consensus. Florida: FT Press.

Rodríguez del Tronco, J.; Rodríguez del Tronco, R. y Vico García, N. (2018). Smart feedback. Conversaciones para el desarrollo del talento. Madrid: Lid Editorial. 
Rodríguez, E. (2010). Estilos de liderazgo, cultura empresarial y eficacia: un estudio empírico en pequeñas y medianas empresas. Revista de Ciencias Sociales, 16(4), 629-641. https://www.redalyc.org/ pdf/280/28016613006.pdf

Rodríguez, E. y Pedraja, L. (2009). Análisis del impacto del proceso de toma de decisiones estratégicas sobre la eficacia de las organizaciones públicas. Innovar, 19 (35), 33-46. https://revistas.unal. edu.co/index.php/innovar/article/ view/28707/34857

Rodríguez-Cruz, Y. (2015). Gestión de Información y del Conocimiento para la toma de decisiones organizacionales. Bibliotecas Anales de la investigación, 11 (11), 150-163. http://revistas.bnjm.cu/index.php/ BAl/article/view/203

Rodríguez-Cruz, Y. y Pinto, M. (2017). Requerimientos informacionales para la toma de decisiones estratégicas en organizaciones de información. Transinformação, 29(2), 175-189. https://doi.org/10.1590/2318$\underline{08892017000200005}$

Rojas Andrade, R. (2013). El liderazgo comunitario y su importancia en la intervención comunitaria. Psicología para América Latina, México, D.F., $n^{\circ}$ 25, pp. 57-76. http://pepsic.bvsalud.org/scielo. php? script $=$ sci arttext\&pid $=$ S1870350X2013000200005

Ruiz González, M. A., Bodes Bas, A., y Dominicis Matas, D. (2016). Metodología de intervención socioeconómica: una experiencia en la empresa cubana. Economía y Desarrollo, 157 (2), 77-90. https://bit. Iy/3yyPO6o

Salgado Roa, J., Lería Dulcic, J. F., Arcos Rico, L. D. C., Pineda Alcayaga, L. C., González Carmona,
C. M. (2018). Actitud y resistencia al cambio organizacional en trabajadores mineros. Revista de Psicología. 36 (1), 105-136. https:// doi.org/10.18800/psico.201801.004

Sánchez Cobarro, P. H. (2016). Organizaciones emocionalmente inteligentes. Revista de Ciencias de la Comunicación e Información, 21, (1), 19-33. http://doi.org/10.35742/ rcci.2016.21(1).19-33

Sánchez Manchola, I. D. (2011). Los estilos de dirección y liderazgo: Propuesta de un modelo de caracterización y análisis. Pensamiento \& gestión, 25, 1-39. https://www.redalyc.org/articulo. oa?id=64612241002

Seligman, M.E.P. y Csikszentmihalyi, M. (2000). Positive psychology: An introduction. American Psychologist, 55, 5-14. http://doi. org/10.1037//0003-066x.55.1.5

Sierra-Caballero, F. (2018). Ciberactivismo y movimientos sociales. El espacio público oposicional en la tecnopolítica contemporánea. Revista Latina de Comunicación Social, 73, 980-990. http://doi.org/10.4185/RLCS-2018$\underline{1292}$

Staat, B. R. (2018), Never Stop Learning: Stay Relevant, Reinvent Yourself, and Thrive. Harvard Business Review Press.

Tavares Teixeira, D. (2019). Diretrizes para uma política de comunicação científica para a Universidade do Estado de Mato Grosso (Brasil). Perspectivas de la Comunicación, 12 (1), 135-156. https://doi.org/10.4067/ $\underline{\text { S0718-48672019000100135 }}$

Thorndike, W. (2012). The Outsiders: Eight Unconventional CEOs and Their Radically Rational Blueprint for Success. Cambridge: Harvard 
Liderazgo y gestión empresarial: modelo de liderazgo global de cambio

Business Review Press.

Valle, L. y García, C. (2019). Impacto del empoderamiento de los empleados en el desempeño organizacional en la industria pesquera. COMPENDIUM: Cuadernos de Economía $y$ Administración, 6 (2), 74-90. https:// doi.org/10.46677/compendium. $\underline{\mathrm{v} 6 \mathrm{i} 2.768}$

Vega-Pérez, J. G., Vargas-Ramos, M. F., Amores-Guevara, P.R. y Arias-Tapia, S. A. (2017). Riesgos psicosociales y la seguridad industrial en las lavanderías textiles del Cantón Pelileo. Revista de Comunicación de la SEECI, 43, 135-149. https://doi. org/10.15198/seeci.2017.43.135-149

Wictor, I. y Anderson, S. (2012). The importance of leadership and vision in born global, Business and Management Research, 1 (1), 13-25 http://doi.org/10.5430/bmr.v1n1p13

Zuckerman, E. (2017). Los gatitos monos al rescate. CIC. Cuadernos De Información y Comunicación, 22, 27-46. https://doi.org/10.5209/ 